\title{
Uma base de dados para compartilhamento de experiências no uso de mapas conceituais no acompanhamento de processos de conceituação
}

\author{
Ítalo Modesto Dutra (Laboratório de Estudos em Educação a Distância/UFRGS) - \\ italodutra@lead.cap.ufrgs.br \\ Stéfano Pupe Johann (Laboratório de Estudos em Educação a Distância/UFRGS) - \\ stefanopj@gmail.com \\ Carlos Augusto Piccinini (Laboratório de Estudos em Educação a Distância/UFRGS) - \\ piccguto@yahoo.com.br \\ Léa da Cruz Fagundes (Laboratório de Estudos Cognitivos/UFRGS) - \\ leafagun@ufrgs.br
}

Resumo: A utilização de um referencial construtivista piagetiano para o uso dos mapas conceituais levou à construção de um modelo de acompanhamento dos processos de conceituação. Esse modelo visa a estabelecer um referencial teórico e metodológico com o objetivo de acompanhar o desenvolvimento dos sistemas conceituais (construção de conceitos) de crianças e jovens no desenvolvimento de Projetos de Aprendizagem na escola. Esse trabalho tem o objetivo de apresentar o design de um banco de dados digital que permita organizar dados de experimentos que usem o modelo de acompanhamento dos processos de conceituação. A construção desse banco de dados permitirá, além de consultas que façam cruzamentos de dados, o compartilhamento com outros pesquisadores interessados e a adaptação do modelo a outros contextos de aprendizagem. Palavras-chave: Banco de Dados, Mapas Conceituais, Construtivismo.

Abstract: The use of a constructivist approach to concept maps led to the construction of a model of analysis of conceptualization processes. This model intends to establish a theoretical and practical approach that aims to follow up the development of youngster's conceptual systems (concept building). This work intends to present the design of a digital database able to organize data of experiments that use the model of analysis to follow up conceptualization processes. The development of this database will allow, besides queries that links significant data, sharing with other researchers and the adaptation of the model to other educational contexts. Keywords: Database, Concept Maps, Construtivism.

\section{Introdução}

Nos últimos anos, a utilização de mapas conceituais na Educação tem tido um crescimento expressivo. Em contraste com um texto escrito, o mapa conceitual parece revelar-se fértil em possibilidades no sentido de uma expressão mais fiel do sistema de significações de um sujeito e, mais ainda, um excelente dispositivo de apoio a novas construções conceituais (um objeto para pensar com).

A técnica de construção e a teoria a respeito dos Mapas Conceituais foi desenvolvida pelo pesquisador norte-americano Joseph Novak (Novak e Gowin, 1984). Ele define mapa conceitual como uma ferramenta para organizar e representar conhecimento. O mapa conceitual é uma representação gráfica em duas dimensões de um conjunto de conceitos construídos de tal forma que as relações entre eles sejam evidentes (figura 1). Os conceitos aparecem dentro de caixas nos nós do grafo enquanto que as relações entre os conceitos são especificadas através de frases de ligação nos arcos que unem os conceitos. A dois ou mais conceitos, conectados por frases de ligação 
criando uma unidade semântica, chamamos de proposição. As proposições são uma característica particular dos mapas conceituais se comparados a outros grafos similares como os mapas mentais.

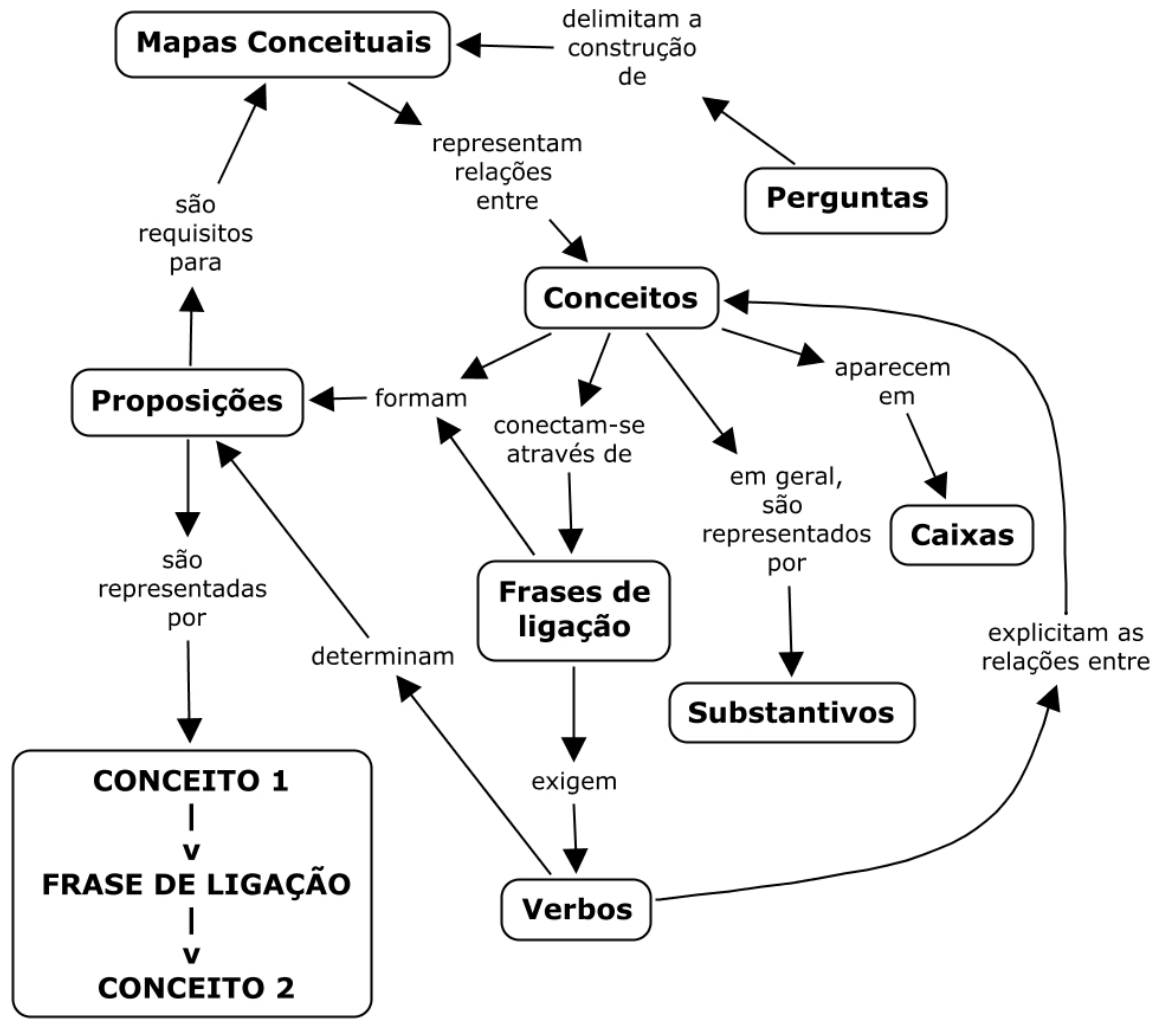

Figura 1. Mapa conceitual sobre mapas conceituais

Nossa proposta (Dutra, Fagundes, Johann e Piccinini, 2006) sugere um modelo de análise que utiliza essa técnica de forma sistemática. Esse modelo foi desenvolvido na orientação de crianças (10 a 12 anos) em idade escolar em seus projetos de pesquisa, o autor solicitou que estas construíssem e revisassem mapas conceituais periodicamente. Também foram realizadas entrevistas com base nesses mapas, além de seminários e rodadas de apresentação. Cada sujeito desenvolveu um diário pessoal (blog) e alguns sites com idéias e conclusões a respeito de sua pesquisa. Partindo de um referencial construtivista, é proposto que esses dados, analisados em conjunto, permitem o acompanhamento do desenvolvimento dos processos de conceituação.

O modelo de análise proposto, portanto, faz uso de diferentes fontes de evidências: mapas conceituais, discurso escrito (textos), discurso transcrito (entrevistas). Além disso, produziu-se também registros das atividades realizadas e do progresso da pesquisa a cada dia. Para armazenar esses dados, foi criado um banco digital (BD) que permite categorizar cada um desses extratos e as respectivas análises realizadas.

Em grande parte, o design do banco de dados a ser apresentado a seguir foi desenvolvido a partir deste primeiro, que comportou apenas o experimento inicial de desenvolvimento do modelo. O objetivo do nosso trabalho é mostrar o design projetado do $\mathrm{BD}$, buscando possibilitar que novos pesquisadores e experimentos tenham acesso aos dados, bem como fazer uso de programação que permita o desenvolvimento de melhorias subsequentes. De maneira geral, o banco de dados pretende servir como uma 
organização do material referente ao uso de mapas conceituais para acompanhamento dos processos de conceituação.

\section{Modelo de análise}

Conforme mencionado anteriormente, o modelo de análise foi desenvolvido a partir de um experimento inicial de orientação e intervenções em um projeto de aprendizagem. Esses projetos consistem, basicamente, em pesquisas realizadas por crianças (com idades entre 10 e 12 anos) durante seu horário escolar. Passaremos a uma breve descrição a respeito desse modelo, para o qual o banco de dados é direcionado.

Nos parece importante destacar o fato de que as atividades realizadas pelos sujeitos do experimento na realização de seus Projetos de Aprendizagem implicavam, em sua grande maioria, a leitura e a elaboração (e conseqüentes revisões) de textos. Mesmo que em alguns momentos fosse possível acessar imagens, gráficos e simulações na internet, que eles mesmos trouxessem algum objeto real (um microchip, uma lâmpada, um prisma, espelhos etc.) ou ainda que nós realizássemos algumas experiências (com circuitos elétricos), a principal fonte de informação estava na forma de texto escrito.

É possível observar que, embora os registros em linguagem natural possam, precocemente, apresentar muitas informações (não necessariamente na forma de proposições) a respeito dos conceitos em construção nas investigações do sujeito, a representação em mapa conceitual "demora" a expressar uma organização mais elaborada. Tal fato é corroborado também por dados dos outros sujeitos do estudo. De certa forma, a restrição imposta para a construção do mapa (o uso das proposições) requer uma reflexão por parte do sujeito gerando tomadas de consciência acerca das relações (possíveis ou disponíveis em seu sistema de significações) entre os conceitos. Assim, os ditos "resumos" dos textos e informações coletadas ou, ainda, os textos reescritos "com as suas próprias palavras" podem levar a uma falsa idéia de que tais informações foram incorporadas aos sistemas de significação do sujeito.

O conjunto de indicadores construídos a partir da revisão teórica (e conseqüente adaptação) oferecem, cada um a seu modo, uma perspectiva de análise das produções dos sujeitos envolvidos no estudo. Dessa forma, a identificação e classificação das operações lógicas formais nas classes e operações proposicionais, dos níveis de implicações entre significações e da elaboração, no discurso em linguagem natural, das classes-objeto fornecem elementos que serviram de base para o acompanhamento dos processos constitutivos das relações nos sistemas de significação dos sujeitos. Faz-se necessário, portanto, uma articulação entre os diferentes elementos obtidos nas análises com a intenção de produzir um conjunto consistente de evidências a respeito dos processos de conceituação em curso.

O modelo de análise e interpretação das produções (discurso em linguagem natural e mapas conceituais) articula construtos teóricos de Jean Piaget (1976,1977 e 1978), Piaget \& Rolando Garcia (1989) e de Jean-Blaise Grize (1996 e 1997)) para lançar luz a respeito de processos que não são acessíveis senão a partir aproximações fornecidas por atos de representação dos sujeitos em questão. Essa articulação constituiu uma adaptação e uma aplicação dos elementos encontrados na teoria desses autores de forma a servir a uma análise de um novo objeto de investigação ou, pelo menos, de um novo enfoque, em especial devido a representação de conhecimento permitida pelos mapas conceituais. 
A construção de mapas conceituais esteve, desde o início, no centro de nossas discussões e representa o principal foco de atenção das análises realizadas. Contudo, isso não significa que o mapa conceitual deva ser considerado de forma isolada do contexto das representações que o sujeito realiza de tal forma que, por si só, ele possa constituir um "produto final" absoluto e fonte de todas as evidências que nós necessitamos. Ainda assim, como pudemos perceber no exemplo ilustrativo apresentado na seção anterior, a construção de mapas conceituais faz diferença no que tange o acompanhamento dos processos de conceituação em curso de um sujeito.

De maneira geral, portanto, o modelo de análise utiliza diversos tipos de recursos e observa-os de maneira específica (figura 2). A lógica natural é uma ferramenta importante para o entendimento de trechos em linguagem escrita, enquanto a lógica proposicional é utilizada para a análise dos mapas conceituais. Uma característica importante, e que marca a construção do banco de dados de forma peculiar, é que o acompanhamento dos processos de conceituação é realizado de maneira dinâmica. Assim, muitas vezes um texto ou um mapa é revisado por seu autor, e essas mudanças são um dos focos de interesse para o pesquisador.

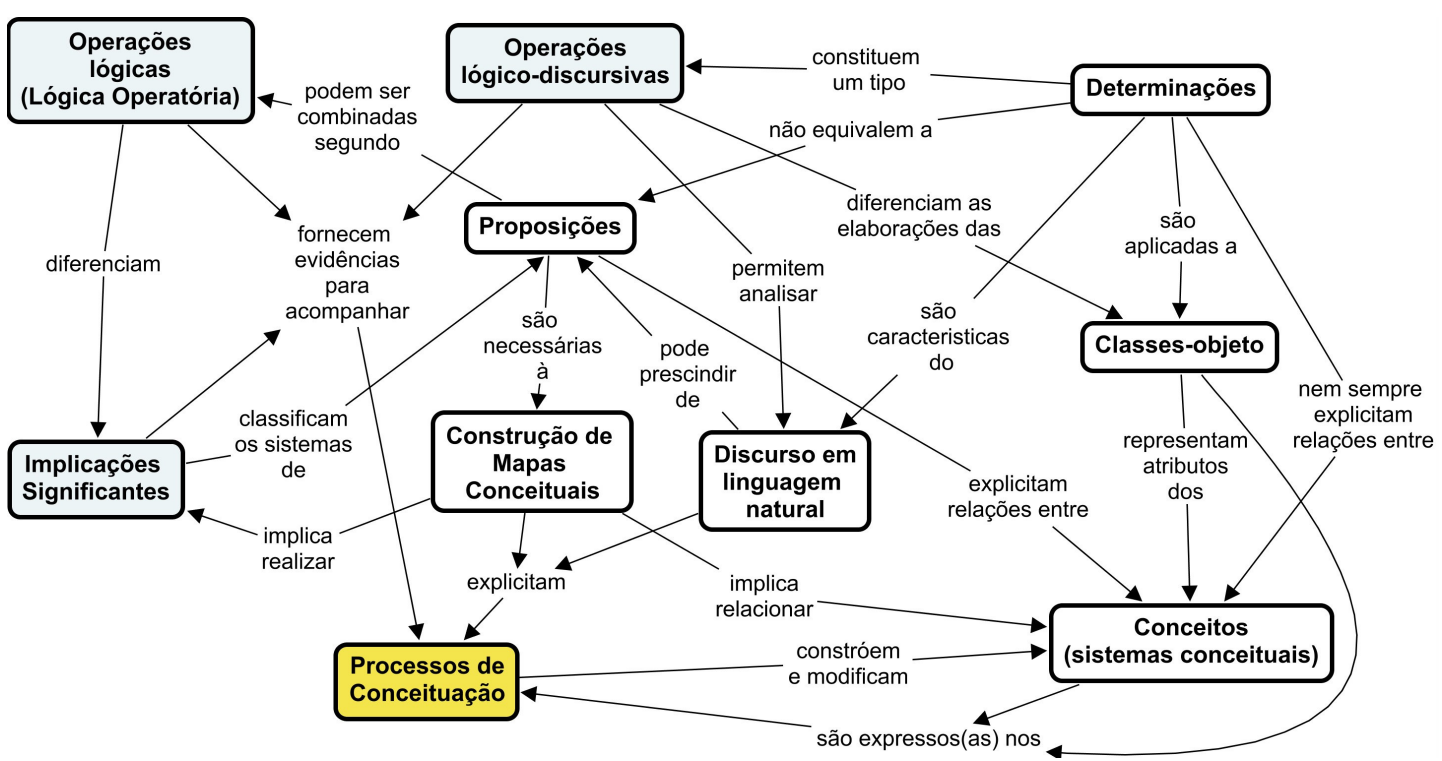

Figura 2. O modelo de acompanhamento de processos de conceituação

\section{Estrutura do banco de dados}

Desenvolvido na plataforma Linux com interface phpMyAdmin, o banco de dados permite exibição e cruzamento de dados de maneira que se adaptasse às necessidades de armazenamento e catalogação do modelo de análise. Assim, o primeiro passo foi uma categorização prévia de cada um dos tipos de dado derivados da experiência obtida durante o estudo inicial (tabela 1).

\begin{tabular}{|c|c|c|}
\hline Tipo de dado & Descrição & $\begin{array}{c}\text { Identificação } \\
\text { (id_tipo) }\end{array}$ \\
\hline Mapa conceitual & $\begin{array}{c}\text { Representação gráfica que explicita } \\
\text { relações entre conceitos; revisada } \\
\text { sistematicamente }\end{array}$ & 1 \\
\hline
\end{tabular}




\begin{tabular}{|c|c|c|}
\hline CINTED-UFRGS & Novas Tecr & na \\
\hline Blog (aluno) & $\begin{array}{l}\text { Diário desenvolvido periodicamente } \\
\text { pelos próprios sujeitos }\end{array}$ & 2 \\
\hline Blog (professor) & $\begin{array}{c}\text { Diário com observações e análises dos } \\
\text { pesquisadores que acompanharam o } \\
\text { projeto }\end{array}$ & 3 \\
\hline Wiki & $\begin{array}{l}\text { Site com conclusões dos sujeitos a } \\
\text { respeito da pesquisa; possui recursos } \\
\text { de versionamento }\end{array}$ & 4 \\
\hline Entrevista clínica & $\begin{array}{c}\text { Entrevista realizada com o objetivo de } \\
\text { obter mais elementos sobre a } \\
\text { construção do mapa conceitual }\end{array}$ & 5 \\
\hline
\end{tabular}

Tabela 1. Descrição e categorização dos dados produzidos.

Cada novo registro, portanto, é armazenado sob um campo (id_registro) e recebe uma identificação adicional, referente à sua categoria (id_tipo) (figura 2). É importante enfatizar que, por enquanto, os mapas conceituais são catalogados sob a forma de texto e de um link para um servidor externo; futuramente, o banco de dados será capaz de suportá-los em formato gráfico.

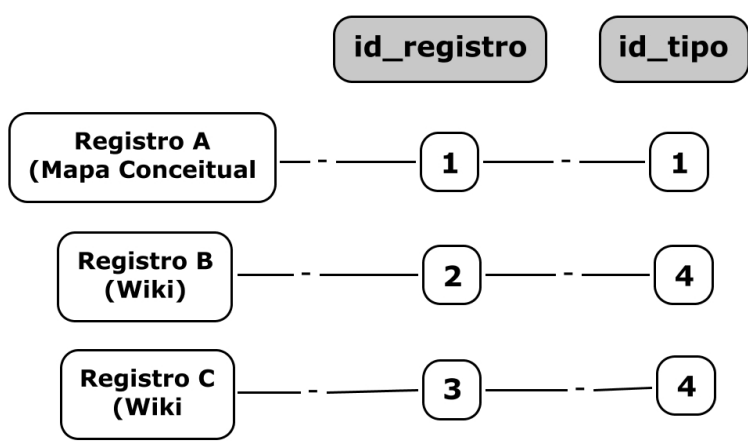

Figura 2: Diagrama que ilustra a associação de cada registro a uma identificação e a uma categoria.

Além dessa categorização inicial, cada registro está associado, também, a dois campos: seção e sujeitos. De maneira geral, esses campos identificam a autoria, a data e o projeto ao qual o registro está ligado. O primeiro (id_seção) recebe uma identificação referente ao campo "experimento" e à data (figura 3). Dessa forma, cada registro deve estar inserido em um contexto definido, o que é fundamental, dada a natureza dinâmica sustentada pelo modelo de análise.

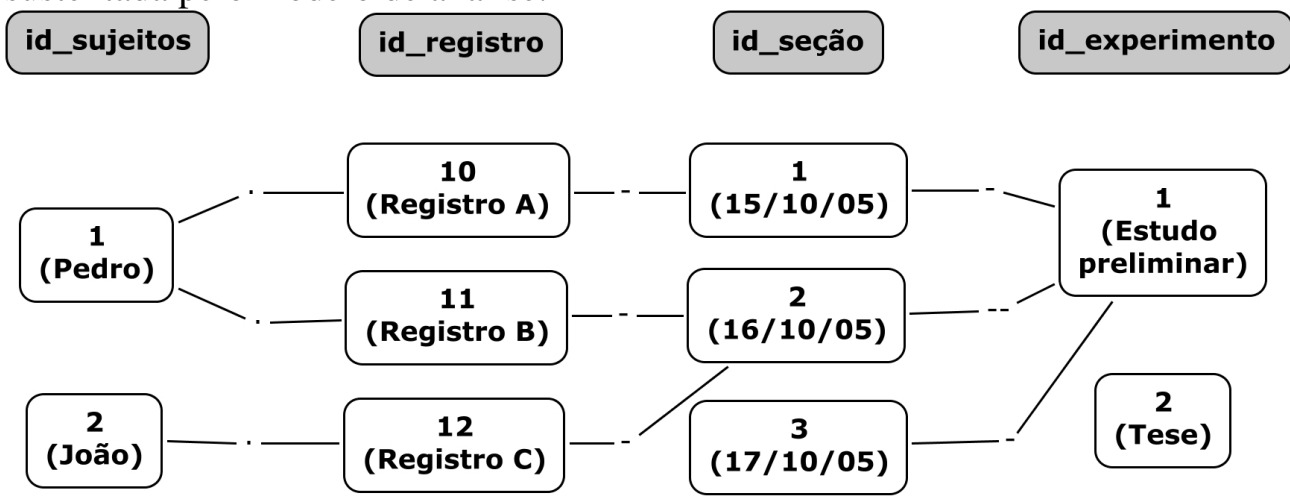

Figura 3: Exemplo da integração entre os diferentes campos de identificação. 
O outro elemento a que os registros estão associados, conforme demonstrado na figura 3, é a identificação de sujeitos. Cada sujeito participante de qualquer experimento recebe uma matrícula, relacionada à função id_experimento. Assim, um sujeito (ou um pesquisador) recebe uma identificação numérica simples no campo id_sujeitos e é matriculado em um ou mais experimentos através da função id_matricula (figura 4)

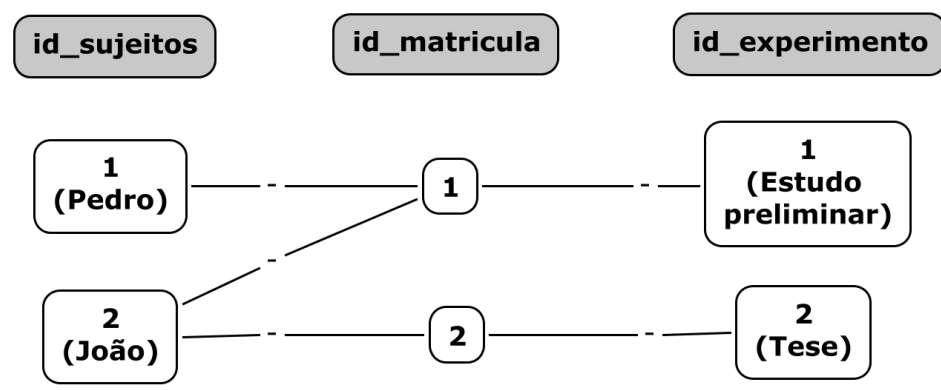

Figura 4: Diagrama referente à associação do campo "sujeitos" a outros elementos.

Recapitulando, o que foi descrito da estrutura, até aqui, capacita o banco de dados a introduzir um registro (id_registro), catalogá-lo (id_tipo), situá-lo (id_seção), atribuir autoria (id_sujeitos) e identificar o experimento a que pertence (id_experimento). Além disso, a função id_matricula cataloga os sujeitos participantes dos experimentos listados.

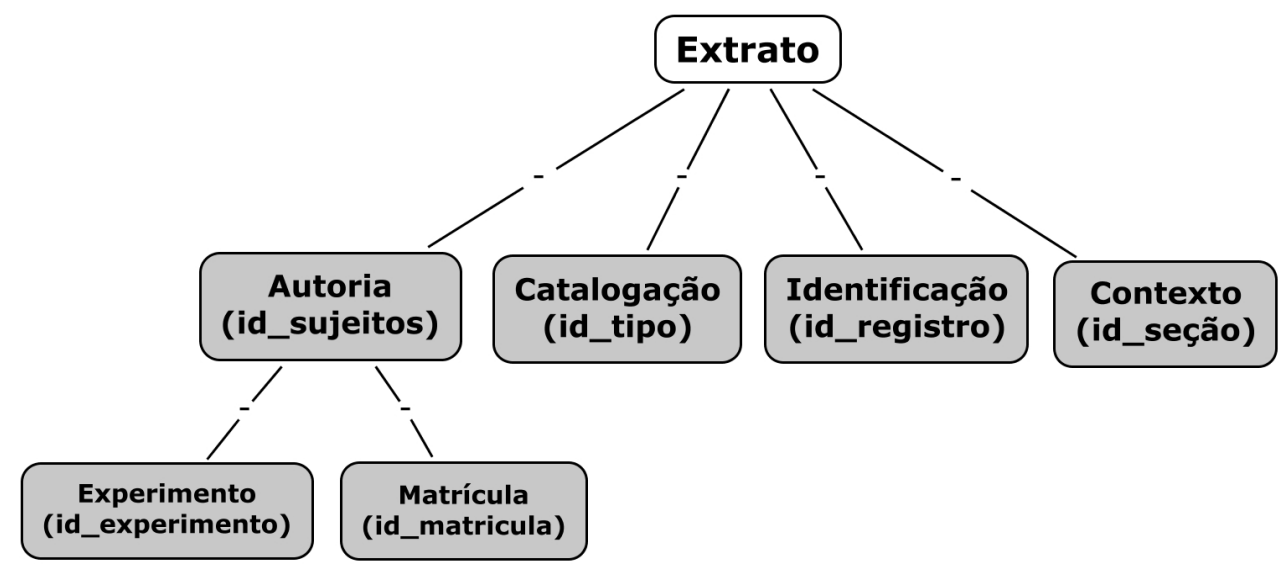

Figura 5: Recapitulação das funções descritas até o momento.

Um recurso a ser implementado diz respeito às categorias de análise. Em princípio, elas serão capazes de se separar em "camadas", de forma que um pequeno extrato (como uma frase ou palavra, catalogada em um dos registros anteriores) possa ser acompanhado por diversas versões e transformações que recebe, mesmo que haja mudança no nome com que é referido pelo sujeito. Assim, torna-se mais simples o acompanhamento de um conceito ou classe-objeto entre diversas elaborações realizadas pelo sujeito.

Nesse sentido, o banco de dados é capaz de armazenar uma diversidade de análises referentes a um mesmo trecho. Tendo por base materiais de diversos tipos 
(mapas conceituais, blogs, entrevistas, etc.), o modelo de análise desenvolvido utilizou três tipos de perspectivas teóricas para entendimento dos dados (Tabela 2)

\begin{tabular}{|c|c|c|}
\hline Tipo de análise & Descrição & $\begin{array}{c}\text { Identificação } \\
\text { (id_analise) }\end{array}$ \\
\hline Implicações significantes & $\begin{array}{c}\text { Apontam evidências do } \\
\text { desenvolvimento lógico apresentado } \\
\text { pelos mapas conceituais }\end{array}$ & 1 \\
\hline Lógica Operatória & $\begin{array}{c}\text { Aplicadas na análise das proposições } \\
\text { elaboradas nos mapas }\end{array}$ & 3 \\
\hline $\begin{array}{c}\text { Operações lógico- } \\
\text { discursivas }\end{array}$ & $\begin{array}{c}\text { Ferramental referente à análise do } \\
\text { discurso escrito e falado }\end{array}$ & 2 \\
\hline
\end{tabular}

Tabela 2. Tipos de análise e sua identificação.

Em suma, o banco de dados é capaz de organizar e cruzar os dados da maneira descrita anteriormente. Aliado a isso, também é possível acompanhar o desenvolvimento conceitual em um contínuo, baseando-se em análises que agrupam os diferentes termos inter-relacionados. A seguir, alguns exemplos que mostram como a estrutura do banco de dados se aplica à utilização prática.

\section{Resultados parciais}

Embora a aplicação de todos os recursos ainda precise de revisões, a estrutura atual do banco de dados permite vislumbrar sua aplicabilidade no contexto em que está inserido. Por enquanto, os dados de dois experimentos de um mesmo pesquisador estão armazenados nele. Os exemplos apresentados a seguir fazem parte desses dois estudos.

Uma das funções mais simples, porém essencial, de um organizador como o proposto neste artigo é a possibilidade de listar diversos tipos de categorias relacionadas. Um estudo longitudinal, por exemplo, se beneficiaria da função que permite a recapitulação de todos os extratos de um mesmo sujeito, ao longo de diversos estudos. A figura 6 demonstra isso, selecionando ainda um só tipo de produção.

Data

d_tipo $=1$ (Mapas Conceituais

id_sujeito $=25$ (Sujeito Y)

id_experimento
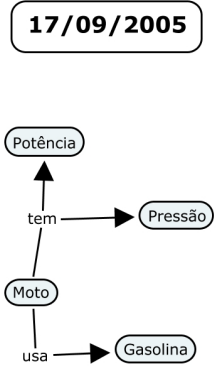

1
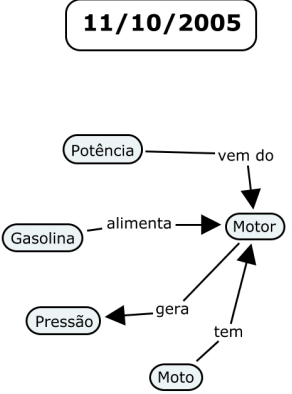

1
$08 / 07 / 2006$

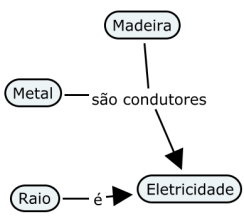

2

Figura 6. Diversos mapas de um mesmo sujeito ao longo de dois estudos.

Outra função importante do banco de dados é mais específica: a capacidade de articular os registros em função de uma análise posterior. Assim, uma categoria de 
análise é capaz de tomar emprestados extratos de diferentes datas, dando sentido às mudanças efetuadas (figura 7).

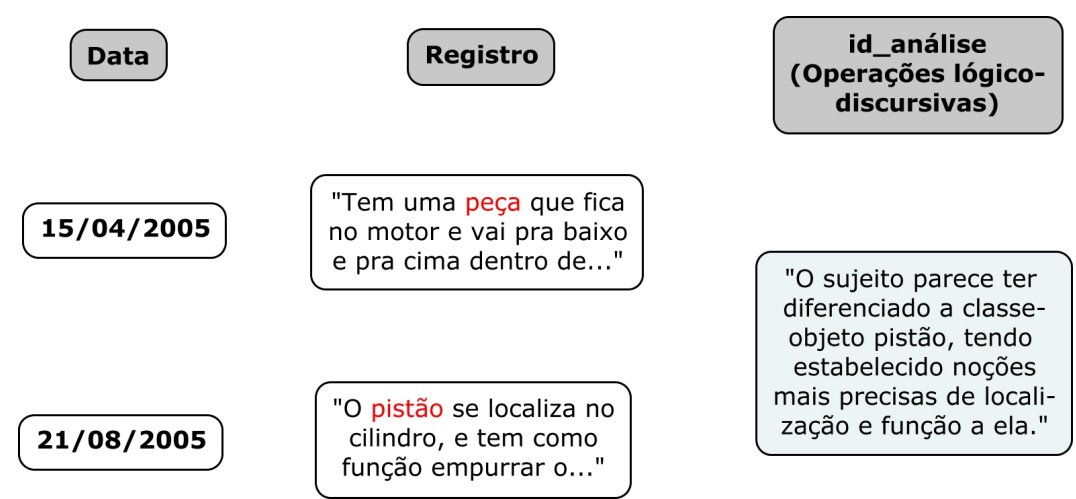

Figura 7. Exemplo da função de busca articulada pela análise.

Muitos outros exemplos de cruzamentos de dados são possíveis: disponibilizar todos os dados que contenham determinado conceito, exibir os registros de apenas dois tipos em ordem cronológica, utilizar sujeitos até determinada idade, etc. Os exemplos demonstrados até aqui demonstram que o banco de dados se mostra capaz de atender as demandas geradas pela utilização do modelo de análise que deu origem a ele. No entanto, nada impede que seja utilizado para outro tipo de perspectiva

O último exemplo, evidenciado na figura a seguir (figura 8), mostra um possível estudo quantitativo baseado nos dados recolhidos durante os experimentos armazenados no banco até aqui. Embora não seja propriamente relacionado à temática para a qual o banco de dados foi idealizado, esse exemplo mostra a utilidade de um recurso capaz de centralizar, organizar e cruzar o material de diversos estudos.

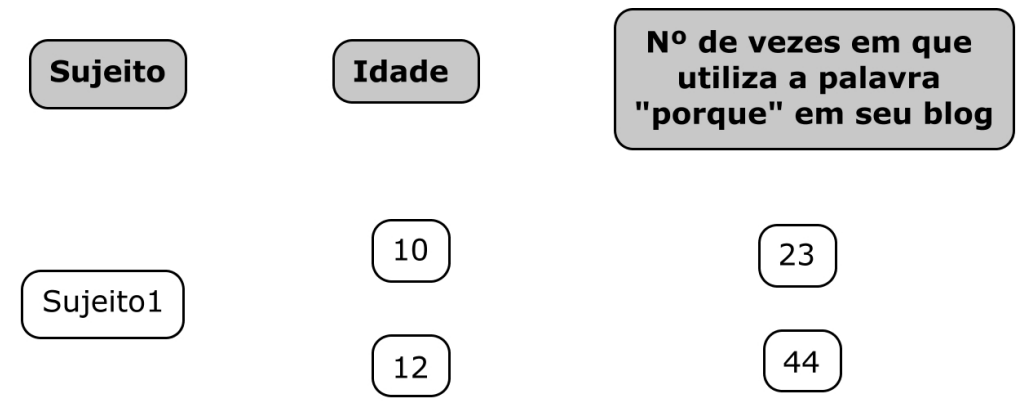

Figura 8. Hipotético estudo quantitativo suportado pelo uso do banco de dados.

\section{Conclusões}

Com a popularização do uso de mapas conceituais na educação e em outras áreas, torna-se cada vez mais importante que novos tipos de armazenamento de dados estejam disponíveis. Estudos mais abrangentes poderão resultar desse tipo de iniciativa, além de maior possibilidade de trocas entre pesquisadores ligados a esse segmento. $\mathrm{O}$ Portal de Mapas Conceituais na Educação, associado ao Laboratório de Estudos em Educação a Distância, é um site com esse objetivo. A comunidade de professores cadastrado nessa comunidade, que já ultrapassa o número de 300 membros, é um exemplo do público-alvo da ferramenta descrita neste artigo. 
Baseado em uma abordagem construtivista aos mapas conceituais, o banco de dados proposto ao longo deste artigo se mostra uma ferramenta capaz de armazenar e organizar os registros gerados pela utilização do modelo de análise de Dutra, Fagundes, Johann e Piccinini (2006). À medida que um maior número de pesquisadores começarem a utilizar esta abordagem, se faz necessária a construção de uma ferramenta capaz de se tornar um espaço de integração e suporte a futuros experimentos. $\mathrm{O}$ banco de dados busca ocupar esse espaço, oferecendo recursos especificamente voltados a esses objetivos.

Em breve, espera-se que o banco de dados já esteja completamente operacional. O próximo passo, então, será disponibilizá-lo para uso pela comunidade interessada. Com base nas experiências trazidas por outros estudos e pesquisadores, o banco de dados poderá ser aperfeiçoado e redesenhado de forma a contemplar contribuições e perspectivas originais.

\section{Referências}

Ausubel, D. P (2000). The Acquisition and Retention of Knowledge: a cognitive view. Dordrecht: Kluwer Academic.

Dutra, Í. M., Fagundes, L. C., Johann, S.P. \& Piccinini, C.A. (2006). Logical systems and natural logic: concept mapping to follow up the conceptualization processes. In: CMC 2006 - Second International Conference on Concept Mapping, 2006, San José - Costa Rica.

Grize, J. B. (1996). Logique naturelle et communication. Paris: PUF.

Grize, J. B. (1997). Psicologia Genética e Lógica. In Banks-Leite, L. (Org.). Percursos Piagetianos. São Paulo: Cortez.

Novak, J. D. \& Gowin, D. B. (1984). Learning How to Learn. New York: Cambridge University Press.

Piaget, J. (/1976). Ensaio de Lógica Operatória. Porto Alegre: Globo.

Piaget, J. (1977). A tomada de consciência. São Paulo: Melhoramentos.

Piaget, J. (1978). Fazer e compreender. São Paulo: Melhoramentos. 
Piaget, J. \& Garcia, R (1987/1989). Hacia una logica de significaciones. Cidade do México: Gedisa. 